\title{
Anticipatory Planning and Control of Grasp Positions and Forces for Dexterous Two-Digit Manipulation
}

\author{
Qiushi Fu, ${ }^{1}$ Wei Zhang, ${ }^{1}$ and Marco Santello ${ }^{1,2}$ \\ ${ }^{1}$ Department of Kinesiology and ${ }^{2}$ School of Biological and Health Systems Engineering, Arizona State University, Tempe, Arizona 85287
}

\begin{abstract}
Dexterous object manipulation requires anticipatory control of digit positions and forces. Despite extensive studies on sensorimotor learning of digit forces, how humans learn to coordinate digit positions and forces has never been addressed. Furthermore, the functional role of anticipatory modulation of digit placement to object properties remains to be investigated. We addressed these questions by asking human subjects (12 females, 12 males) to grasp and lift an inverted T-shaped object using precision grip at constrained or self-chosen locations. The task requirement was to minimize object roll during lift. When digit position was not constrained, subjects could have implemented many equally valid digit position-force coordination patterns. However, choice of digit placement might also have resulted in large trial-to-trial variability of digit position, hence challenging the extent to which the CNS could have relied on sensorimotor memories for anticipatory control of digit forces. We hypothesized that subjects would modulate digit placement for optimal force distribution and digit forces as a function of variable digit positions. All subjects learned to minimize object roll within the first three trials, and the unconstrained device was associated with significantly smaller grip forces but larger variability of digit positions. Importantly, however, digit load force modulation compensated for position variability, thus ensuring consistent object roll minimization on each trial. This indicates that subjects learned object manipulation by integrating sensorimotor memories with sensory feedback about digit positions. These results are discussed in the context of motor equivalence and sensorimotor integration of grasp kinematics and kinetics.
\end{abstract}

\section{Introduction}

Dexterous object manipulation is learned through the formation and retrieval of sensorimotor memories generated by previous hand-object interactions (Johansson and Westling, 1984,1988 a), thus allowing the modulation of digit forces in an anticipatory fashion, i.e., before the object is lifted (Gordon et al., 1993; Burstedt et al., 1999; Salimi et al., 2000). Anticipating, rather than reacting to, the effect of a given object property, e.g., slip or tilt, leads to a more efficient grasp control than reflexdriven corrective force responses (Johansson and Westling, 1987, 1988a,b).

Object manipulation is achieved by generating net forces and torques that are appropriate for a desired behavioral outcome, e.g., a compensatory torque at the onset of the manipulation to prevent roll when lifting an object with an asymmetrical center of mass (Salimi et al., 2000). It should be emphasized that net forces and torques can be modulated not only by changing the neural drive to hand muscles, but also by applying digit forces at differ-

Received July 21, 2009; revised May 14, 2010; accepted May 24, 2010.

This publication was made possible by Bioengineering Research Partnership Grant R01-NS050265 from the National Institute of Neurological Disorders and Stroke (NINDS) at the National Institutes of Health (NIH) and Collaborative Research Grant BCS-0819547 from the National Science Foundation (NSF). Its contents are solely the responsibility of the authors and do not necessarily represent the official views of NIH, NINDS, or NSF. We thank Dr. Andrew Gordon, Dr. Miya Rand, and Dr. Jamie Lukos for their comments on an earlier version of the manuscript, and two anonymous reviewers for their insightful comments.

Correspondence should be addressed to Marco Santello, Department of Kinesiology, Arizona State University, Tempe, AZ 85287-0404. E-mail: marco.santello@asu.edu.

DOI:10.1523/JNEUROSCI.4159-09.2010

Copyright $\odot 2010$ the authors $\quad 0270-6474 / 10 / 309117-10 \$ 15.00 / 0$ ent locations on the object (Lukos et al., 2007). Therefore, the coordination between digit forces and positions is critical for successful manipulation. However, no previous studies have investigated how subjects learn to control both variables through consecutive manipulations. Specifically, most previous research has focused on how subjects learn digit force modulation by grasping an object at fixed locations often constrained by the position of force sensors. Conversely, studies that examined the modulation of digit placement as a function of task or object properties have not measured the concurrent modulation of individual digit forces (Cohen and Rosenbaum, 2004; Friedman and Flash, 2007; Lukos et al., 2007, 2008; Ciocarlie et al., 2009). Therefore, it is unknown how the removal of digit placement constraints on an object affects digit force control.

It should be emphasized that allowing subjects the choice of digit placement enables them to explore a wider range of relations between digit forces and positions, which has been speculated to lead to a more optimal digit force distribution (Lukos et al., 2007). At the same time, the removal of digit placement constraints might result in significant trial-to-trial variability of digit position. Consequently, reliance on sensorimotor memories of digit forces from previous trials for anticipatory grasp control might not be sufficient to attain a consistent performance. This is because the points of force application in the current trial might be very different from those used in previous trials, thus requiring a digit force distribution that has not been previously experienced.

To determine the extent to which choice of digit placement affects anticipatory force control, we asked subjects to grasp an 


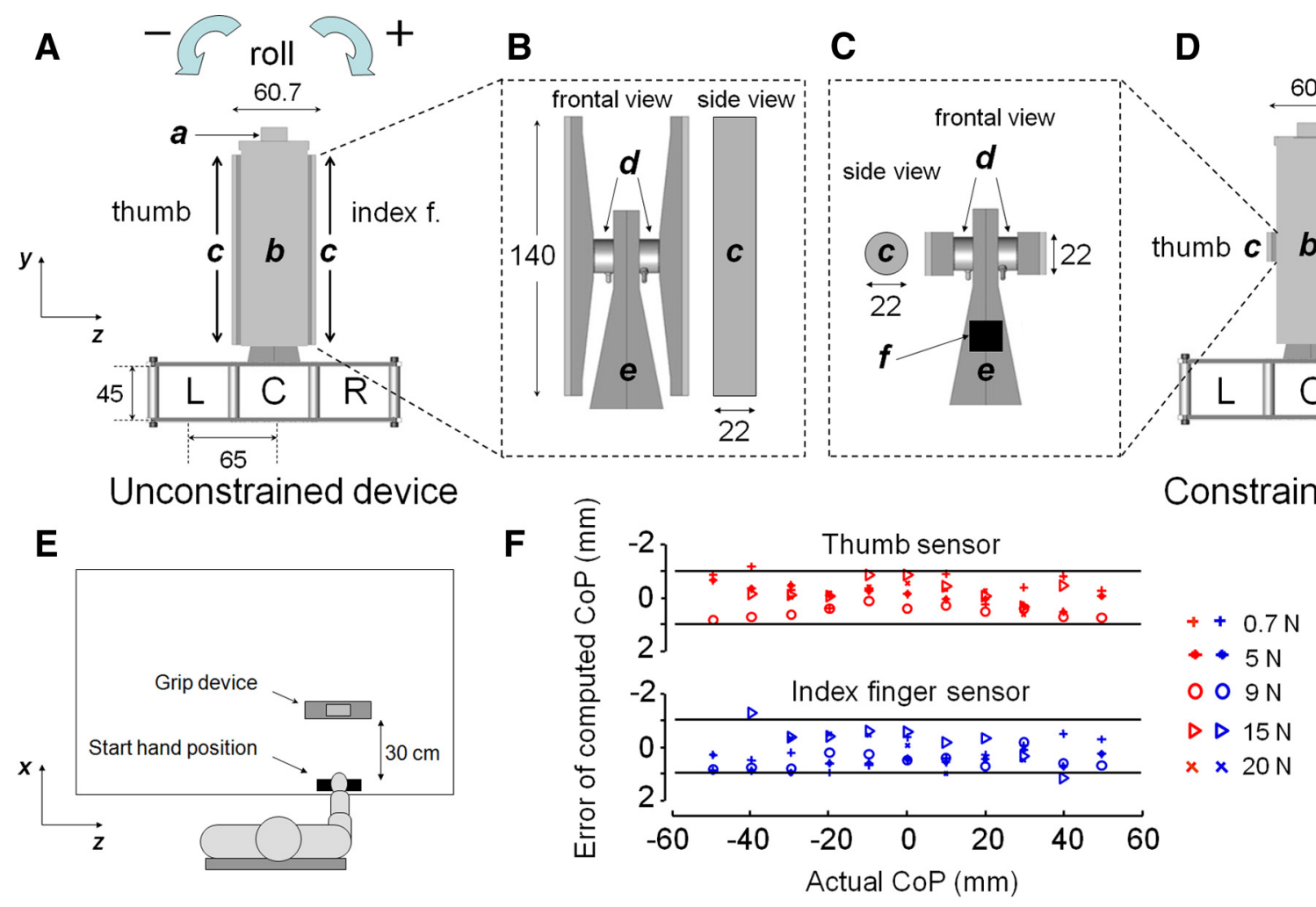

Figure 1. Experimental setup. $\boldsymbol{A}-\boldsymbol{D}$, The two custom-built grip devices used for the study. The unconstrained device allowed subjects to choose digit placement on two long, graspable surfaces $(\boldsymbol{A}, \boldsymbol{c})$, whereas the constrained device could only be grasped on two small, graspable surface $(\boldsymbol{D}, \boldsymbol{c})$. For both grip devices, the graspable surfaces were mounted on force/torque sensors $(\boldsymbol{B}, \boldsymbol{C}, \mathrm{d})$ mounted on either side of a central block $(B, C, e)$. The force/torque sensors measured the $x-, y$-, and $z$-components of forces and torques applied by the thumb and index finger. A magnetic tracker $(A, D$, a) was mounted on the top of each grip device to measure its position and orientation. Two panels $(A, D, b)$ were mounted on the front and back of each grip device to block the sensors from view. A light mass $(50 \mathrm{~g} ; C, f)$ was added to the constrained device for the purpose of matching the weight of both devices. Units of the dimensions of the grip device components are in millimeters. $A, D, A$ mass $(400 \mathrm{~g})$ was added to the left, center, or right slots at the bottom of the device ( $L$, $C$ and $R$, respectively). The dimensions of the slot prevented motion of the mass during object movement. Object rolls toward the thumb and finger sides were defined as negative and positive angles, respectively, relative to the vertical $\left(0^{\circ}\right)$ in the gravitational frame of reference. $E$, The position of the subject's hand relative to the object before reach onset (top view; figure is not to scale). $\boldsymbol{F}$, The difference (error) between the computed and actual center of pressure of loads of different magnitudes applied perpendicular to the graspable surface mounted on the thumb and index finger sensors of the unconstrained device. The center of pressure of each load was computed using the force and torque outputs of each sensor.

object with and without digit placement constraints while minimizing object roll during lift. The grip devices were designed to quantify trial-to-trial learning and execution of the anticipatory control of both digit positions and forces. We hypothesized that subjects would learn to modulate digit positions to optimize the distribution of digit forces and would modulate digit forces to compensate for trial-to-trial variability of digit position to attain a consistent grasp performance.

\section{Materials and Methods}

Subjects. Twenty-four right-handed subjects (12 females and 12 males, ages 20-26 years) with normal or corrected-to-normal vision took part in the experiments. The subjects had no history of musculoskeletal or neurological disorders. All subjects were naive to the experimental purpose of the study and gave informed consent to participate in the experiment. The experimental procedures were approved by the Institutional Review Board at Arizona State University and were in accordance with the Declaration of Helsinki.

Experimental apparatus. We asked subjects to reach, grasp, lift, and replace one of two custom-made inverted T-shaped grip devices consisting of a vertical block attached to a horizontal base (Fig. $1 A, D$ ) using the thumb and index finger of their right hand. The only difference between the two devices was the dimension of the graspable surfaces. Specifically, the graspable surfaces of one of the grip devices consisted of two long parallel PVC bars (length $=140 \mathrm{~mm}$; width $=22 \mathrm{~mm}$ ) (Fig. $1 \mathrm{~A}, B$ ). For the second grip device, the graspable surfaces consisted of two collinear circular plates (diameter $=22 \mathrm{~mm})($ Fig. $1 C, D)$, similar to those of grip devices used by previous studies of two-digit grasping (e.g., Salimi et al., 2000, 2003; Bursztyn and Flanagan, 2008). Therefore, one grip device allowed subjects to choose digit placement anywhere along the vertical graspable bars (unconstrained device) (Fig. 1A), whereas the other constrained digit placement at fixed locations on the object (constrained device) (Fig. 1D). For both grip devices, the horizontal distance between the two graspable surfaces was $60.7 \mathrm{~mm}$. Each graspable surface was mounted on a force/torque transducer (Fig. 1B,C) (see Data recording below for details). The locations of the sensors relative to the graspable surfaces were blocked from view by two panels (Fig. $1 A, D$ ) to prevent visual cues that might have biased choice of digit placement in the unconstrained group.

The center of mass (CM) of the object could be changed across blocks of trials by adding a mass $(400 \mathrm{~g})$ in one of three slots at the base of the object (Fig. 1 $A, D$ ). The external torques resulting from the added mass with respect to the CM of the unloaded grip device were $-255,0$, and 255 $\mathrm{N} \cdot \mathrm{mm}$ when the mass was added at the left, center, or right slot, respectively. Note that throughout the text the definitions of "left" and "right" $\mathrm{CM}$ locations refer to the mass added on the thumb and index finger side of the grip device, respectively. For both grip devices, the total mass of the object (grip device plus added mass) was $0.796 \mathrm{~kg}$. The difference between the weights of the graspable surfaces of the two grip devices was eliminated by placing a $50 \mathrm{~g}$ of mass in the middle of the object (Fig. 1C).

Experimental task. Subjects were assigned to one of two groups $(n=12$ for each group). The unconstrained group used the apparatus with long graspable surfaces and the constrained group used the apparatus with small circular graspable surfaces. Both subject groups were given the same task instructions. 


\section{Unconstrained group}
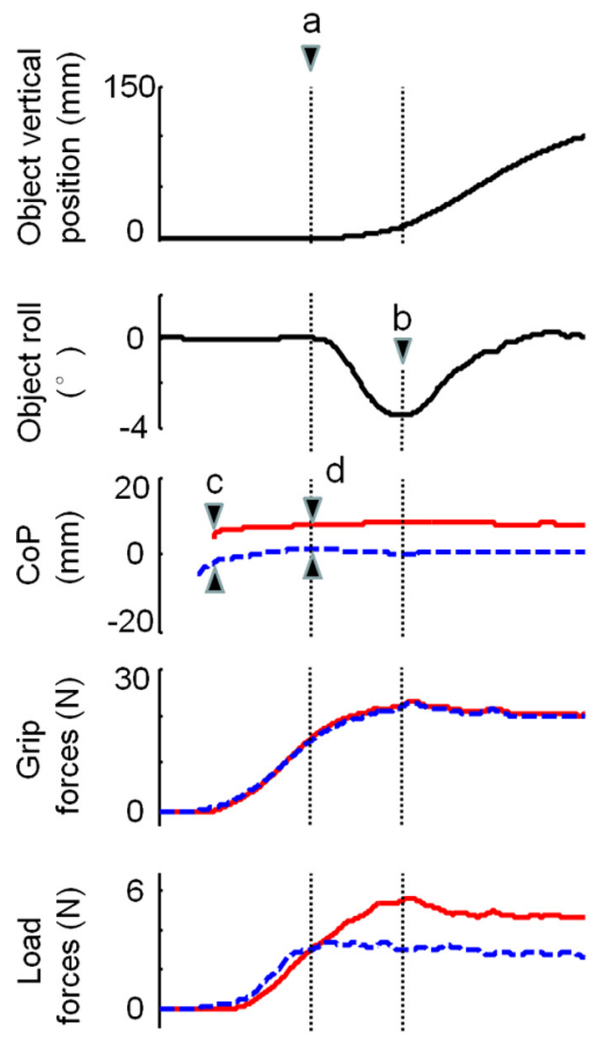

Time
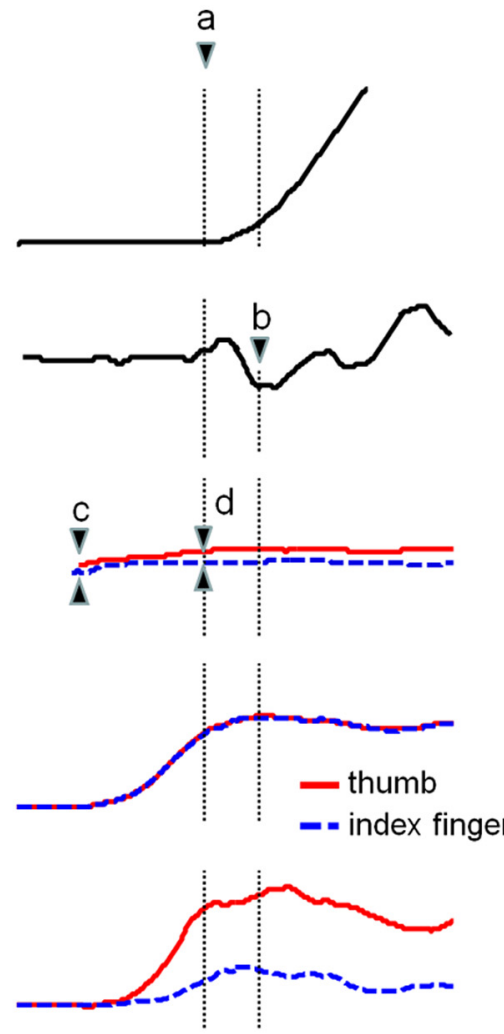

Time

Figure 2. Experimental variables. The experimental variables analyzed in our study are shown for each time epoch from digit contact to object hold for one representative trial performed with the unconstrained and constrained grip device (left and right column, respectively). Data are from two representative subjects. From top to bottom, traces are object vertical position, object roll, thumb and index finger $\mathrm{CoP}_{\mathrm{P}}$ grip forces, and load forces. Time epochs and variables shown are object lift onset (a), peak object roll (b), early digit contact (c), and vertical distance between thumb and index finger CoPs at object lift onset (d). The vertical coordinate of digit $\mathrm{COP}$ is defined as positive or negative when it is above or below, respectively, the center $(y=0)$ of the sensor. Data are from the fifth object lift with the mass added to the left of the object. Peak object roll was minimized to a similar extent by both subjects. However, note that at object lift onset, the subject grasped the unconstrained grip device by placing the thumb higher than the index finger and exerted similar load forces with both digits. Even though the subject in the constrained group placed the thumb slightly higher than the index finger, a much larger load force with the thumb than the index finger was used.

The apparatus was placed on the table at a distance of $30 \mathrm{~cm}$ from the hand start position and aligned with the subject's right shoulder, the object's and subject's frontal planes being parallel to each other (Fig. $1 E$ ). The subject sat on a height-adjustable chair with the wrist resting on a table, the forearm pronated, the arm oriented in the parasagittal plane passing through the shoulder, and the right hand in the designated start position. Subjects started the reach movement after a verbal signal from the experimenter. Instructions to the subjects were as follows: (1) to reach, grasp, lift, and replace the object at a natural speed; (2) to grasp the object only with the thumb and index fingertips and only on the graspable surfaces; (3) to lift the object vertically to a comfortable height of 15-20 cm above the table while trying to maintain its vertical alignment, i.e., to minimize object roll; (4) to hold it for $\sim 1 \mathrm{~s}$; and (5) to replace it on the table. Subjects were aware of that they could grasp anywhere on the graspable surfaces while avoiding the edges. As the object was located at chest height, subjects had full view of the object and their hand, as well as where they grasped the object.

We also asked subjects to extend the middle, ring and little fingers during the grasp to prevent these fingers from exerting force on either the object or the index finger. Before starting data collection, the experimenter demonstrated the task, after which subjects performed three practice trials. For both the demonstration and the practice trials, the mass was added to the center slot of the grip device. The practice trials

\section{$0.2 \mathrm{~s}$}

were used to allow subjects to become familiarized with object weight and friction. During data collection, one of the experimenters visually verified that subjects complied with all of the above instructions on each trial.

After practice trials, subjects performed three blocks of 10 consecutive trials per CM location for a total of 30 experimental trials. Although subjects could not anticipate CM location at the beginning of each block of trials (i.e., trial 1), they were informed that CM location would remain the same for the entire block of trials. Changes of object CM across blocks of trials were performed out of view to prevent subjects from anticipating object CM location. The consecutive presentation of a given object CM location was used to allow subjects to gain information (implicit learning) about the magnitude and direction of the external torque caused by the added mass (Lukos et al., 2007, 2008). This, in turn, allowed us to quantify the time course of trial-to-trial learning of anticipatory control of digit forces and position. The order of CM blocks of trials was counterbalanced across subjects. On average, the time between each trial and blocks of trials was $10 \mathrm{~s}$ and $1 \mathrm{~min}$, respectively.

Data recording. Forces and torques exerted by the thumb and index finger were recorded by two 6-axis force/torques sensors (ATI Nano-17 SI50-0.5, ATI Industrial Automation; force range: 50,50 , and $70 \mathrm{~N}$ for $x$-, $y$-, and $z$-axes, respectively; force resolution: $0.012 \mathrm{~N}$; torque range: $500 \mathrm{~N} \cdot \mathrm{mm}$; torque resolution: $0.063 \mathrm{~N} \cdot \mathrm{mm}$ ) (Fig. $1 B, C$ ). A magnetic tracker (Fastrack, Polhemus) was fixed on the top of the vertical block (Fig. $1 A, D$ ) to record the position and orientation of the object. Force and torque data were recorded through two analog-to-digital converter boards (PCI-6220 DAQ, National Instruments; sampling rate, $1 \mathrm{kHz}$ ), whereas position data were recorded through a serial port (sampling rate, $120 \mathrm{~Hz}$ ). Collection of force and position data were synchronized using custom software (LabView, National Instruments).

Data processing. After data collection, position data were resampled at the same sampling rate of the force data, after which both data were run though a fifth-order Butterworth low-pass filter (cutoff frequency, $30 \mathrm{~Hz}$ ). For data processing and analysis, we wrote custom software in MATLAB (Mathworks) to compute the following variables: (1) grip force and load force were defined as the normal and tangential components of each digit force exerted at the digit center of pressure with respect to the graspable surfaces; (2) digit center of pressure $(\mathrm{CoP})$ was defined as the vertical coordinates of the center of pressure of the contact between the finger pad and the graspable surface (Fig. $1 A, D$ ) relative to the origin of the sensor frame of reference corresponding to its center. Digit CoP was calculated from the force and torque components measured by the force/torque sensor relative to its frame of reference. Calibration of each sensor with its graspable surface revealed that the $x$ - and $y$-coordinates of each digit center of pressure could be computed with an accuracy of $\pm 1.2 \mathrm{~mm}$ (Fig. $1 F$ ) above a threshold of $0.75 \mathrm{~N}$ in normal force. Positive and negative CoP values denote CoP above and below the center of the sensor, respectively (note that the center of the sensor corresponds to the center of the graspable surfaces) (Fig. $1 B, C$ ); (3) object roll was defined as the angle between the gravity vector and the vertical axis of the object within the frontal plane of the object. Positive and negative values denote clockwise and counterclockwise rolls, i.e., toward the index finger and thumb side, respectively (Fig. 1 $A$ ); (4) performance variables. We used digit force and 


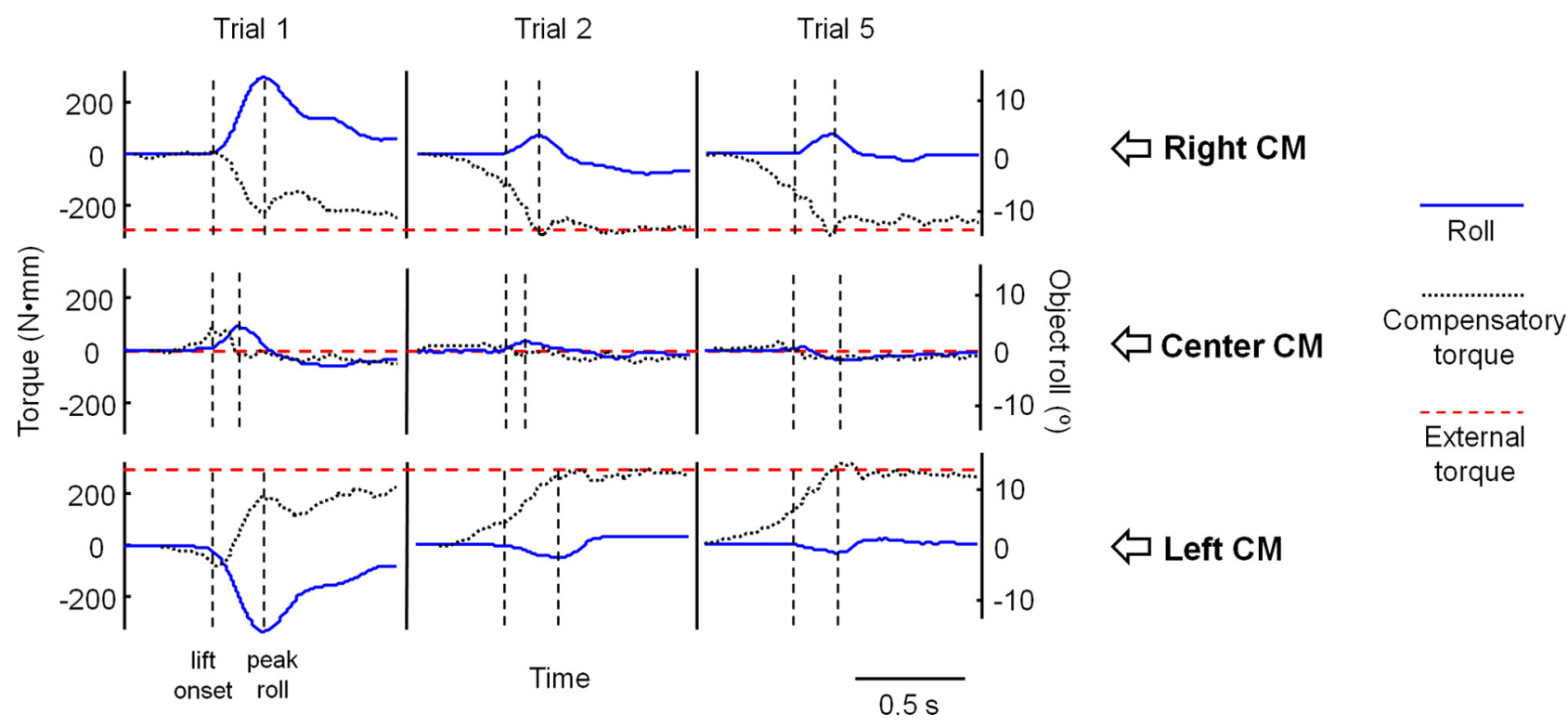

Figure 3. Time course of object roll and compensatory torque. Top to bottom rows show the time course of object roll (solid line), compensatory torque (dotted line), and external torque (dashed horizontal line) from right, center, and left CM conditions, respectively, for one representative subject (subject no. 6). Left to right columns show data from trials 1,2 , and 5 obtained from the unconstrained grip device. To facilitate visual comparison between the external and compensatory torques, both torques are plotted with the same sign even though their signs are opposite. Object lift onset and peak object roll are denoted by the first and second dashed vertical lines, respectively.

CoP data to compute the following performance variables: $(a)$ the average of the digit grip forces $\left(F_{\mathrm{GF}}\right),(b)$ the difference between thumb and index finger load forces $\left(d_{\mathrm{LF}}\right)$, and $(c)$ the vertical distance between thumb and index finger $\operatorname{CoP}\left(d_{\mathrm{y}}\right)$. These three variables are necessary to produce the compensatory torque for minimizing object roll, i.e., balancing the external torque caused by the added mass. Specifically, load force distribution $d_{\mathrm{LF}}$ acts on the width between two graspable surfaces resulting in a load force torque, whereas $F_{\mathrm{GF}}$ acts on digit placement $d_{\mathrm{y}}$, producing a normal force torque. The compensatory torque results from the sum of load force torque and normal force torque (see supplemental material for more details, available at www.jneurosci.org).

The aforementioned variables were computed at the following time points: (1) digit early contact was defined as the time at which the grip force of both digits crossed a threshold of $0.75 \mathrm{~N}$ and remained above it for $300 \mathrm{~ms}$ (Fig. 2). This criterion ensured accurate estimation of digit CoPs at early contact, hence the initial positioning of the digits; (2) object lift onset was defined as the time at which the vertical position of the grip device crossed a threshold of $0.5 \mathrm{~mm}$ and remained above it for $400 \mathrm{~ms}$ (Fig. 2). This is the time before subjects could perceive and react to the external torque. Because the present study was designed to examine anticipatory control of grasping, most of the following analyses focused on the time of object lift onset; and (3) peak object roll was defined as the maximum initial object roll occurring within $\sim 250 \mathrm{~ms}$ after object lift onset (Fig. 2). Peak object roll results from erroneous anticipatory control of fingertip forces and/or contact positions before corrective responses to counter object roll can be made at reaction time latencies (for more details, see Lukos et al., 2007).

Statistical analysis. We performed linear regression analysis to quantify the functional role of compensatory torque modulation at object lift onset and peak object roll during the lift. To quantify the time course of learning the relations between digit CoPs and forces, we performed repeated-measures ANOVA with "CM location" (left, center, and right) and "trial" (trial 1 through 10) as within-subject factors, and "group"(data from the unconstrained vs constrained device) as between-subject factors on digit CoPs, forces, and compensatory torque. Note that for the analyses of anticipatory control of digit CoPs and forces, we focused on these variables measured at object lift onset. Comparisons of interest exhibiting statistically significant differences $(p<0.05)$ were further analyzed using post hoc tests with Bonferroni corrections.

We found that subjects approached a stable level of performance (object roll minimization) within the first three trials, after which no further improvement occurred. To quantify anticipatory control of digit forces and positions after learning had occurred, we performed a second set of analyses that focused on trials 4 through 10. ANOVA and post hoc tests were performed on digit position and forces to assess the effects of $\mathrm{CM}$ location and group across the last seven trials within each CM condition. The effects of these two factors were also tested on the variability (SDs of means computed over the last seven trials) of compensatory torque, digit position, and forces. For these analyses, we used a logarithmic transformation to normalize the SDs.

We also examined the correlation between $d_{\mathrm{y}}, F_{\mathrm{GF}}$, and $d_{\mathrm{LF}}$ over the last seven trials of each CM trial block. Before computing Pearson's correlation coefficients $(r)$, we normalized each of these three variables for each subject by removing the mean of the last seven trials from the value of each trial and dividing the result by the SD of the mean. Comparisons between pairs of correlation coefficients were performed on Fisher's $z$-transformed coefficients.

\section{Results}

\section{Compensatory torque and peak object roll}

Figure 3 shows the time course of object roll and compensatory torque from one representative subject grasping and lifting the unconstrained device for each object CM location. For successful object roll minimization to occur, subjects had to learn to match the external torque with a compensatory torque of equal magnitude and opposite direction before the object is lifted (note that, for graphical purposes, in Fig. 3 the external torque is plotted with the same sign as the compensatory torque). Therefore, subjects have to anticipate, rather than react to, the external torque. Such anticipation could not occur on trial 1 since CM location is unknown to the subject, as shown by the little or no compensatory torque exerted on the object before lift onset for any of the three CM object location (Fig. 3, left column). As a result, for the right and left CM conditions (Fig. 3, top and bottom rows, respectively) the object undergoes a large roll $\left(\sim \pm 15^{\circ}\right)$. For the center CM condition (Fig. 3, middle row), the added mass does not create an external torque on the object, and therefore only a small object roll $\left(<5^{\circ}\right)$ during object lift occurs.

However, subjects learned to compensate for asymmetric mass distribution and object roll following a single trial (Fig. 3, 
A Unconstrained group
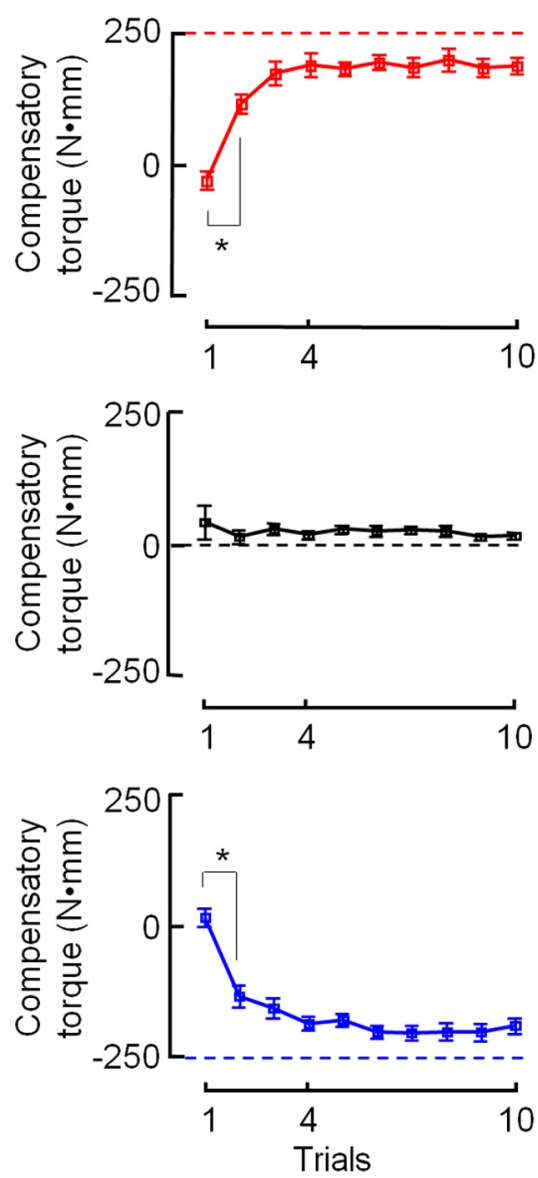

Left CM
B
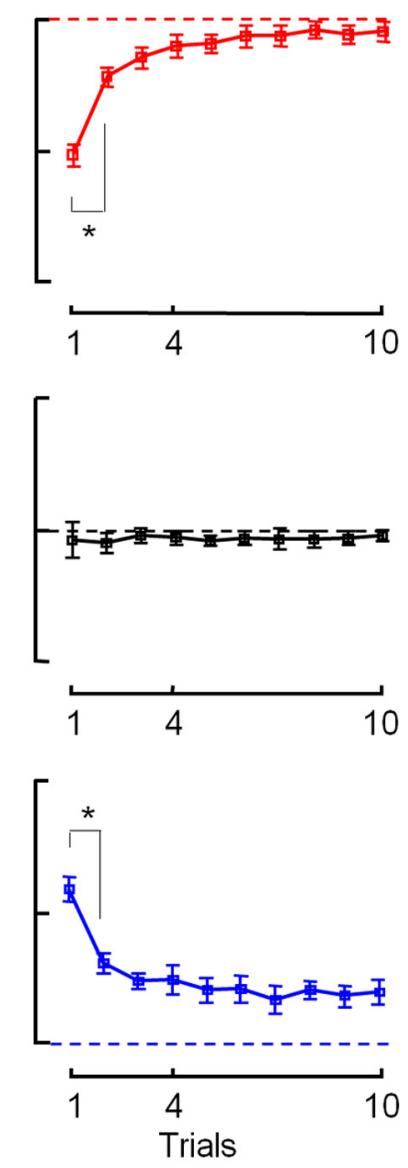

Center CM Right CM

Figure 4. Anticipatory control of compensatory torque as a function of trial. $A, B$, The performance curves of compensatory torque at lift onset for each object CM location as a function of trial averaged across all subjects ( \pm SE) for the unconstrained and constrained group, respectively. Dashed horizontal lines denote the external torque caused by the added mass (note that the external and compensatory torques are plotted using the same convention used for Fig. 3). Asterisks indicate significant differences $(p \leq 0.05)$ between trials.

trials 2 and 5, middle and right columns, respectively). This was accomplished by generating a compensatory torque in the direction opposite to that caused by the added mass (right and left $\mathrm{CM}$ ), starting at $\sim 400 \mathrm{~ms}$ before object lift onset. Consequently, a significant reduction in peak object roll (within $\pm 3^{\circ}$ ) is observed in these trials relative to trial 1.

The compensatory torque produced at object lift onset was a good predictor of the grasp performance, i.e., peak object roll, for both subject groups and all CM conditions. This was confirmed by a strong linear correlation between compensatory torque and peak object roll with an $r$ value of 0.89 ( $p<0.001$; data pooled across all trials, subjects, CM locations, and grip devices). Therefore, we used compensatory torque as a measure of learning anticipatory grasp control for object roll minimization throughout the rest of the manuscript.

\section{Learning of compensatory torque}

Compensatory torque generation (i.e., roll minimization) was learned equally well within the first three trials by both subject groups (no significant interaction, group $\times$ trial) (Fig. 4). Therefore, here we describe data from the unconstrained group only. In trial 1, subjects exerted little or no compensatory torque (mean $\pm \mathrm{SE}$, $-26.6 \pm 16.9,-14.1 \pm 33.8$, and $22.5 \pm$ $17.5 \mathrm{~N} \cdot \mathrm{mm}$ for left, center, and right $\mathrm{CM}$, respectively). This large, between-subject variability in the compensatory torques on the first trial reflect idiosyncratic preferences in how each subject chose digit force and CoPs distributions in response to the unpredictability of object CM. Note also that, unlike the compensatory torques developed after trial 1, the direction and magnitude of these torques are not correctly scaled to the external torque.

However, in trials 2 and 3, the compensatory torque gradually approached the external torque and settled at a mean value of $-188.3( \pm 13.9)$ and 189.7 $( \pm 17.0) \mathrm{N} \cdot \mathrm{mm}$ (right and left $\mathrm{CM}$, respectively) for trials $4-10$, i.e., $\sim 30 \%$ smaller than the external torque (Fig. $4 A$, horizontal dashed lines). The compensatory torque at lift onset for the center CM condition changed little after trial 1, reaching a mean value of $11.3( \pm 13.1)$ $\mathrm{N} \cdot \mathrm{mm}$. The learning curves of compensatory torque of both groups were consistent with the learning curve of peak object roll reported by previous literature (e.g., Salimi et al., 2000).

Despite the fact that subjects' performances were different for center versus left and right CM locations (significant $\mathrm{CM} \times$ trial interaction, $F_{(18,396)}=38.67$, $p<0.001)$, post hoc comparisons performed between neighboring trials (1 vs 2, 2 vs 3 , etc.) revealed that subjects learned to generate anticipatory compensatory torque to minimize object roll early in the trial sequence (Fig. 4) with the only significant difference in compensatory torque occurring between trials 1 and 2 ( $p<0.05$ for both right and left CM in both groups).

Although subjects learned to generate compensatory torque equally well, the underlying compensatory mechanisms differed between two subject groups. Figure 2 shows data from the fifth lift by a representative subject from each group. The subject in the unconstrained group placed the thumb CoP higher than the index finger CoP, thus exhibiting a greater $d_{\mathrm{y}}$ at object lift onset than the subject from the constrained group. In contrast, the subject from the constrained group exerted a larger load force with the thumb than the index finger, thus revealing a much larger $d_{\mathrm{LF}}$ than the subject from the unconstrained group. $d_{\mathrm{y}}$, $d_{\mathrm{LF}}$, and $F_{\mathrm{GF}}$ contribute to the magnitude and direction of the compensatory torque (see supplemental material, available at www.jneurosci.org). Therefore, we present the analyses of each of these three variables.

\section{Learning of digit positions}

The CoP of thumb and index finger at object lift onset were modulated as a function of trial and object CM location. Figure $5 A$ shows $d_{\mathrm{y}}$ averaged across all subjects as a function of trial for 
each CM location and subject group. On the first trial, subjects tended to position the digits collinear to each other regardless of CM location. After trial 1, when lifting the object during the center $\mathrm{CM}$ condition, thumb and index finger CoP tended to remain collinear across all subsequent trials in both groups. In contrast, left and right CM locations elicited opposite patterns of digit CoP modulation. Specifically, the thumb CoP tended to be positioned progressively higher or lower relative to the index CoP for the left and right $\mathrm{CM}$ locations, respectively, and for both subject groups (significant $\mathrm{CM} \times$ trial interaction, $F_{(18,396)}=18.34, p<0.001$; no group $\times$ trial interaction, $p>0.05)$. Similar to the above results on compensatory torque, post hoc comparisons performed between neighboring trials revealed that the only significant change in $d_{\mathrm{y}}$ occurred between trials 1 and 2 but only for right and left CM in both groups $(p<0.05)$.

In addition to measuring the digit $\mathrm{CoP}$ vertical distance at object lift onset, we also examined the digit CoP vertical distance at early contact (see Materials and Methods). The separation of the thumb and index finger occurs at initial contact, suggesting that the hand has been rotated during reach in the direction opposite to the anticipated torque (unconstrained group) (Figs. 2, 5B). Furthermore, the digits do not seem to move vertically on the contact plates after initial contact. Statistical analysis of digit CoP at early contact revealed a very similar pattern to that described above for digit CoP vertical distance at object lift onset (Fig. 5B), i.e., a significantly different CoP distribution as a function of object CM location as a function of practice (significant $\mathrm{CM} \times$ trial interaction, $F_{(18,396)}=6.62, p<$ 0.001). Post hoc comparisons between neighboring trials revealed that the only significant change in $d_{\mathrm{y}}$ at early contact occurred between trials 1 and 2 for the right $\mathrm{CM}$ conditions in the unconstrained group $(p<0.05)$. For the left $\mathrm{CM}$, there was a significant effect of trial within the left CM condition in the unconstrained group $(p<0.05)$ but no significant difference between adjacent trials. However, the center CM condition in the unconstrained group and all CM conditions in the constrained group did not elicit a significant modulation of $d_{\mathrm{y}}$ at early contact $(p>0.05)$. Note that the digit CoPs at early contact are more variable than those at lift onset (Fig. 5), suggesting that some further repositioning occurs in both subject groups as grip and load forces are applied before lift.

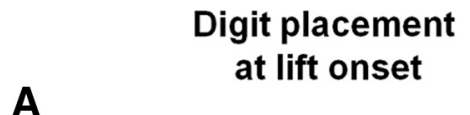

A
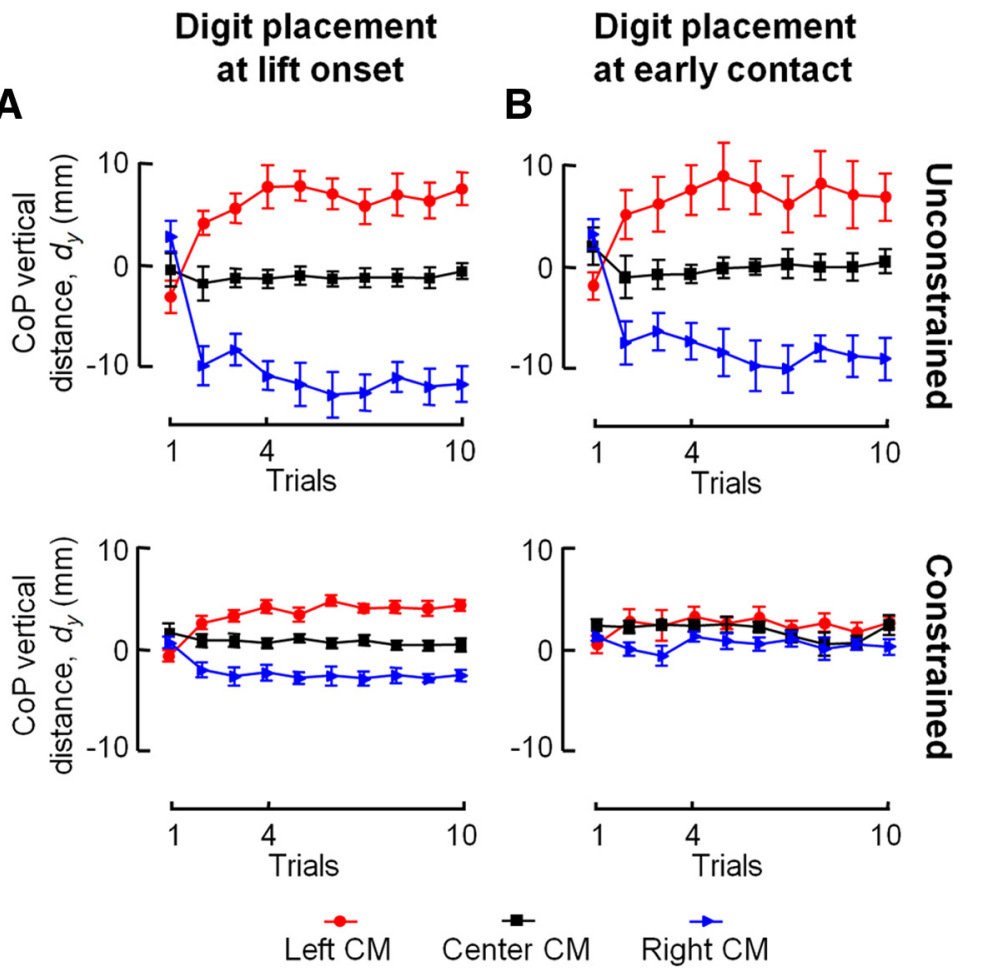

Figure 5. Learning of digit placement. $A, B, d_{\mathrm{y}}$ as a function of trial at object lift onset and early contact, respectively. Data shown in top and bottom rows are from the unconstrained and constrained subject groups, respectively. All data are averages of all subjects ( $\pm \mathrm{SE})$.
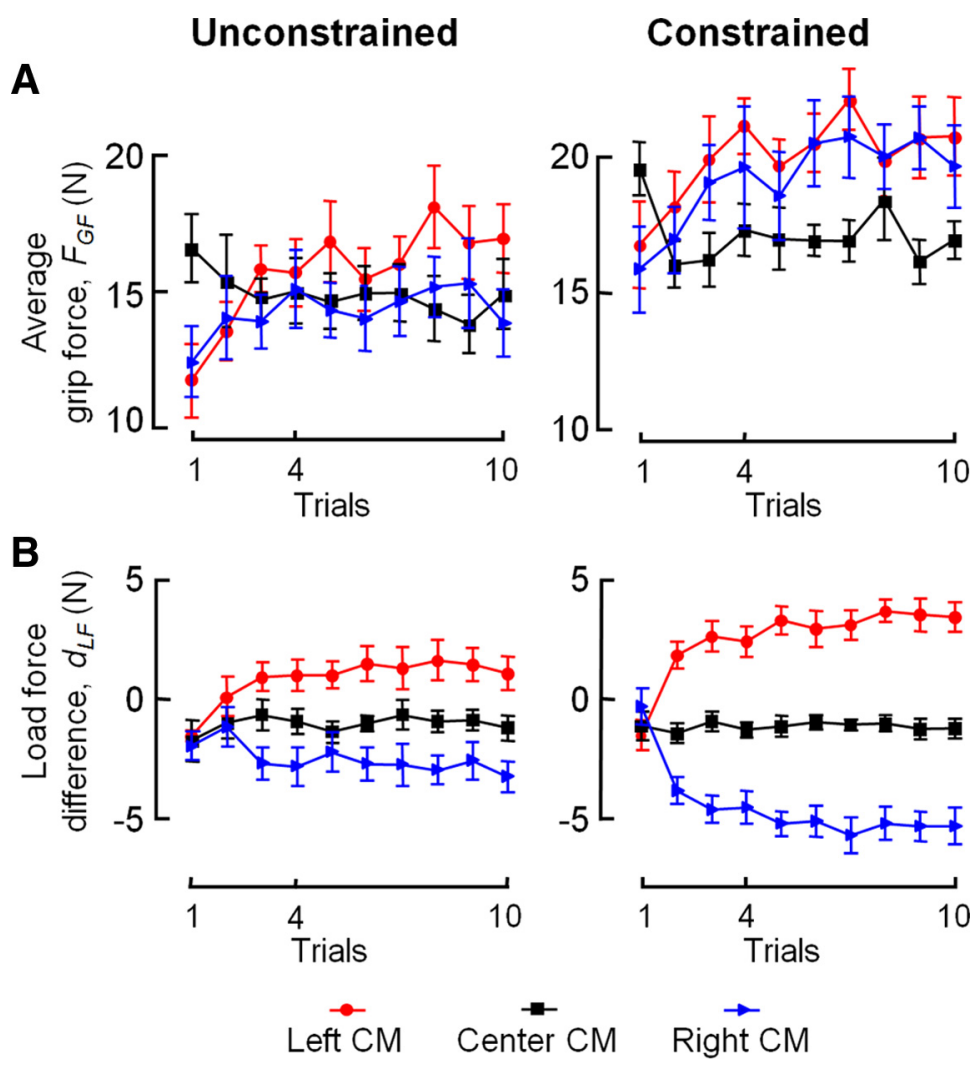

Figure 6. Learning of digit forces. $\boldsymbol{A}, \boldsymbol{B}$, Averaged $F_{\mathrm{GF}}(\boldsymbol{A})$ and $d_{\mathrm{LF}}(\boldsymbol{B})$ at object lift onset as a function of trial and object $\mathrm{CM}$ location for the unconstrained and constrained subject group (left and right columns, respectively). All data are averages of all subjects ( $\pm \mathrm{SE}$ ). 
A

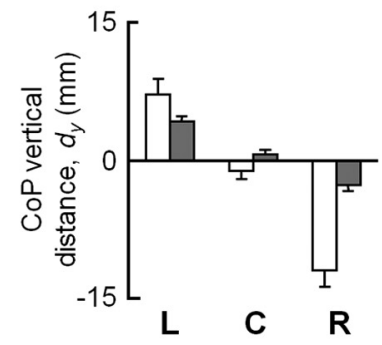

B

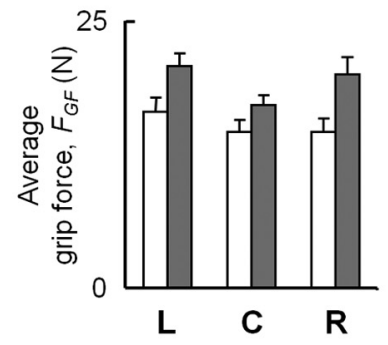

C

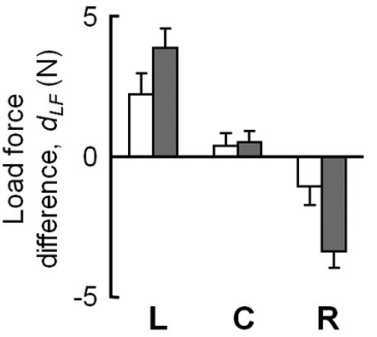

$\square$ Unconstrained $\square$ Constrained

Although the constrained group also showed digit position modulation to object CM, this modulation was significantly smaller than that exhibited by the unconstrained group in left and right CM conditions (three-way ANOVA on factors group, $\mathrm{CM}$, and trial; $\mathrm{CM} \times$ group interaction, $F_{(2,44)}=15.94, p<0.001$; post hoc tests on group effects within right $\mathrm{CM}, p<$ 0.05; nonsignificant group effects within left CM) (Fig. 7A, Table 1). Another important observation is that subjects exhibited a larger modulation of $d_{\mathrm{y}}$ for right than left $\mathrm{CM}$, but only in the unconstrained group ( post hoc tests on CM effects, $p<0.05$ ).

The constrained group used larger grip

\section{Learning of digit forces}

In both subject groups, $F_{\mathrm{GF}}$ tended to increase as a function of trial for left and right $\mathrm{CM}$ conditions and decrease for center CM (significant $\mathrm{CM} \times$ trial interaction, $F_{(18,396)}=4.09, p<0.001 ;$ no group $\times$ trial interaction, $p>0.05$ ) (Fig. $6 A$ ). However, post hoc analyses showed that these trends were significant only for the left CM condition in both groups $\left(F_{(9,99)}=9.08, p<0.001\right.$ and $F_{(9,99)}=2.82, p<0.005$ for unconstrained and constrained groups, respectively).

Subjects also used different patterns of load force distribution across CM locations (Fig. 6B). When lifting the object in the center CM condition, thumb and index finger load forces remained symmetrical across all trials in both subject groups.

In contrast, $d_{\mathrm{LF}}$ tended to be modulated as a function of trial early in the trial sequence and then remain relatively constant for lateral CM conditions (left and right) for both subject groups. On the first trial, subjects tended to use nearly symmetrical load forces for both CM and subject groups. After trial 1, load forces applied by the thumb and index finger were applied asymmetrically to counteract the CM asymmetries. Specifically, the thumb load force tended to be progressively larger or smaller relative to the index load force for the left and right CM conditions, respectively, in both subject groups (significant $\mathrm{CM} \times$ trial interaction, $F_{(18,396)}=11.12, p<0.001 ;$ no group $\times$ trial interaction, $p>$ $0.05)$. However, post hoc comparisons between neighboring trials revealed that only the constrained group modulated $d_{\mathrm{LF}}$ significantly from trials 1 to 2 for both CM conditions (both $p<0.05$ ).

\section{Digit placement and forces during stable performance (trials 4-10)}

All subjects attained a stable level of performance within the first three trials (Fig. 4). Therefore, trial 3 was used as the cutoff, after which (trials 4-10) we defined subjects' performance as stable, i.e., the trial after which further practice did not lead to statistically significant improvements in compensatory torque at object lift onset and object roll minimization. Therefore, the following analyses focused on both magnitude and variability of digit forces and CoP during the last seven trials of each block.

The two groups showed significant differences in overall strategy with constrained trials focusing almost entirely on grasp kinetics (force application), and unconstrained trials relying primarily on kinematics (hand placement on the object). Furthermore, subjects from the constrained group used higher grip force than subjects from the unconstrained group. Although the amplitude of force application and digit alignment differed between groups, the overall direction and resultant forces (net compensatory torque) applied by the two groups were similar. force than the unconstrained group across trials 4-10 (three-way ANOVA on factors group, CM, and trial; main effect of CM, $F_{(2,44)}=5.53, p<0.01$; main effect of group, $F_{(1,22)}=9.47, p<$ 0.001 ) (Fig. 7B, Table 1). Post hoc tests also revealed that subjects used significantly larger grip force only for left and right CM conditions $(p<0.05)$.

As expected, the constrained group showed larger asymmetry of digit load forces than the constrained group in left and right $\mathrm{CM}$ conditions (significant $\mathrm{CM} \times$ group interaction; $F_{(2,44)}=$ 9.24, $p<0.001$; post hoc tests on group effects within left and right CM, both $p<0.05$ ) (Fig. 7C, Table 1). Similar to the differences between the two CM conditions in the extent of digit CoP modulation, subjects exhibited a larger modulation of $d_{\mathrm{LF}}$ for left than right CM ( post hoc tests on CM effects within the unconstrained group; $p<0.05$; nonsignificant $\mathrm{CM}$ effects within constrained group).

\section{Production of compensatory torques: covariation of digit $\mathrm{CoPs}$ and digit forces under different $\mathrm{CoP}$ variability}

The above analysis revealed that digit forces and positions at object lift onset were controlled differently depending on whether or not the grip device constrained digit placement. Surprisingly, however, subjects from the unconstrained and constrained groups learned to generate compensatory torques with similar consistency (Fig. 4, Table 1). This was confirmed by a lack of a significant group effect on the SD of the net result of $d_{\mathrm{y}}, F_{\mathrm{GF}}$, and $d_{\mathrm{LF}}$, i.e., the mean compensatory torque averaged from trials 4 to $10(p>0.05)$. This result is remarkable particularly when considering that the variability of digit placement at object lift onset of the unconstrained group was much larger than the constrained group (Fig. 5A). With regard to $\mathrm{SD}$ of individual digit CoP, we found only a significant main effect of group $\left(F_{(1,22)}=\right.$ $60.96, p<0.001)$. The SD of $d_{\mathrm{y}}$ was significantly different across subject groups and $\mathrm{CM}\left(F_{(1,22)}=26.64\right.$ and $F_{(1,22)}=10.34$, respectively; both $p<0.001)$.

In contrast, there was no significant difference between the two groups with regard to the SD of either digit load forces or grip forces $(p>0.05)$. Recall that the two grip devices share the same mechanics and that $T_{\text {com }}$ is the net result of $d_{\mathrm{y}}, F_{\mathrm{GF}}$, and $d_{\mathrm{LF}}$. Therefore, the large variability in digit placement in the unconstrained group was effectively compensated by digit force modulation such that trial-totrial variability of $T_{\text {com }}$ was similar to the constrained group.

The significantly different variability in digit placement between the unconstrained and constrained subject groups raised the question of how subjects modulated, on a trial-to-trial basis, digit forces as a function of position. We addressed this question by performing linear regression analyses on data normalized to 
Table 1. Summary of performance variables across trials $4-10$

\begin{tabular}{lrrlr}
\hline & \multicolumn{1}{c}{$d_{\mathrm{y}}(\mathrm{mm})$} & \multicolumn{1}{c}{$d_{\mathrm{LF}}(\mathrm{N})$} & \multicolumn{1}{l}{$F_{\mathrm{GF}}(\mathrm{N})$} & \multicolumn{1}{c}{$T_{\text {com }}(\mathrm{N} \cdot \mathrm{mm})$} \\
\hline Left CM & & & & \\
$\quad$ Unconstrained & $7.1 \pm 1.7$ & $2.3 \pm 0.7$ & $16.6 \pm 1.3$ & $189.7 \pm 17.0$ \\
$\quad$ Constrained & $4.2 \pm 0.6$ & $3.9 \pm 0.7$ & $20.7 \pm 1.2$ & $201.6 \pm 16.2$ \\
$\begin{array}{l}\text { Center CM } \\
\quad \text { Unconstrained }\end{array}$ & $0.7 \pm 0.5$ & $-0.4 \pm 0.4$ & $14.6 \pm 1.1$ & $11.3 \pm 13.1$ \\
$\quad$ Constrained & $-1.1 \pm 0.9$ & $0.5 \pm 0.4$ & $17.1 \pm 0.9$ & $-7.8 \pm 12.3$ \\
Right CM & & & & \\
$\quad$ Unconstrained & $-11.8 \pm 1.8$ & $-1.1 \pm 0.7$ & $14.6 \pm 1.2$ & $-188.3 \pm 13.9$ \\
$\quad$ Constrained & $-2.6 \pm 0.7$ & $-3.4 \pm 0.6$ & $20.0 \pm 0.6$ & $-172.1 \pm 18.9$ \\
\hline
\end{tabular}

Data are mean values $( \pm S E)$ of distance between digit center of pressure $\left(d_{\mathrm{y}}\right)$, difference between digit load forces $\left(d_{\mathrm{LF}}\right)$, average grip force $\left(F_{\mathrm{GF}}\right)$, and compensatory torque $\left(T_{\text {com }}\right)$ averaged across trials $4-10$ for each center of mass condition and subject group.

zero mean and unit SD (see Materials and Methods). Most importantly, we observed significant negative correlations between $d_{\mathrm{y}}$ and $d_{\mathrm{LF}}$ in both the unconstrained group $(r=-0.615, p<$ $0.001)$ and constrained group $(r=-0.263, p<0.001)$. Furthermore, the correlation coefficient of the unconstrained group was significantly larger than that of the constrained group $(p<$ 0.001). We also found that center CM was different from left and right $\mathrm{CM}$ conditions, therefore we tested the correlation between $d_{\mathrm{y}}$ and $d_{\mathrm{LF}}$ on center and lateral CMs separately.

For the center $\mathrm{CM}$ condition, both subject groups showed negative correlations between $\mathrm{d}_{\mathrm{y}}$ and $d_{\mathrm{LF}}$ (Fig. 8C,D). This correlation was significantly larger in the unconstrained than in the constrained group $(p<0.05)$.

For left and right CM conditions, the constrained group did not exhibit a significant correlation anymore (Fig. $8 \mathrm{~B}$ ). In contrast, negative correlations were still found for the unconstrained group (Fig. $8 A$ ). Lastly, the strength of the correlation between $d_{\mathrm{y}}$ and $d_{\mathrm{LF}}$ was significantly larger in the unconstrained than in the constrained group $(p<0.05)$. We found no significant correlation between $d_{\mathrm{y}}$ and $F_{\mathrm{GF}}$ (Fig. $\left.8 E, F\right)$ or between $d_{\mathrm{LF}}$ and $F_{\mathrm{GF}}$ in either subject group.

\section{Discussion}

Our task required subjects to lift an object while minimizing roll caused by an external torque due to asymmetric mass distribution. Consistent with previous studies of implicit learning of grasping within blocked trials, subjects learned to minimize object roll within the first three trials by changing digit placement (Lukos et al., 2007, 2008) and/or altering force distribution applied by the fingers (Salimi et al., 2000). The compensatory torque generated by the subjects developed from object contact through lift onset, its magnitude approaching that of the external torque (Fig. 3). Despite considerable trial-to-trial variability of digit positions, subjects learned to minimize the variability of the compensatory torque by modulating digit forces as a function of the digit positions. Interestingly, removal of digit placement constraints did not affect the rate of learning of the compensatory torque even though subjects implemented different relations between digit positions and forces when grasping the two grip devices (Figs. 5 and 6). These results are discussed in relation to the neural mechanisms underlying the sensorimotor integration of grasp kinematics and kinetics.

Functional significance of anticipatory modulation of digit placement and forces for object manipulation

To compensate for the torque generated by asymmetric mass distribution in the test objects, subjects could have altered the applied grip force, load force, and/or hand position on the object.
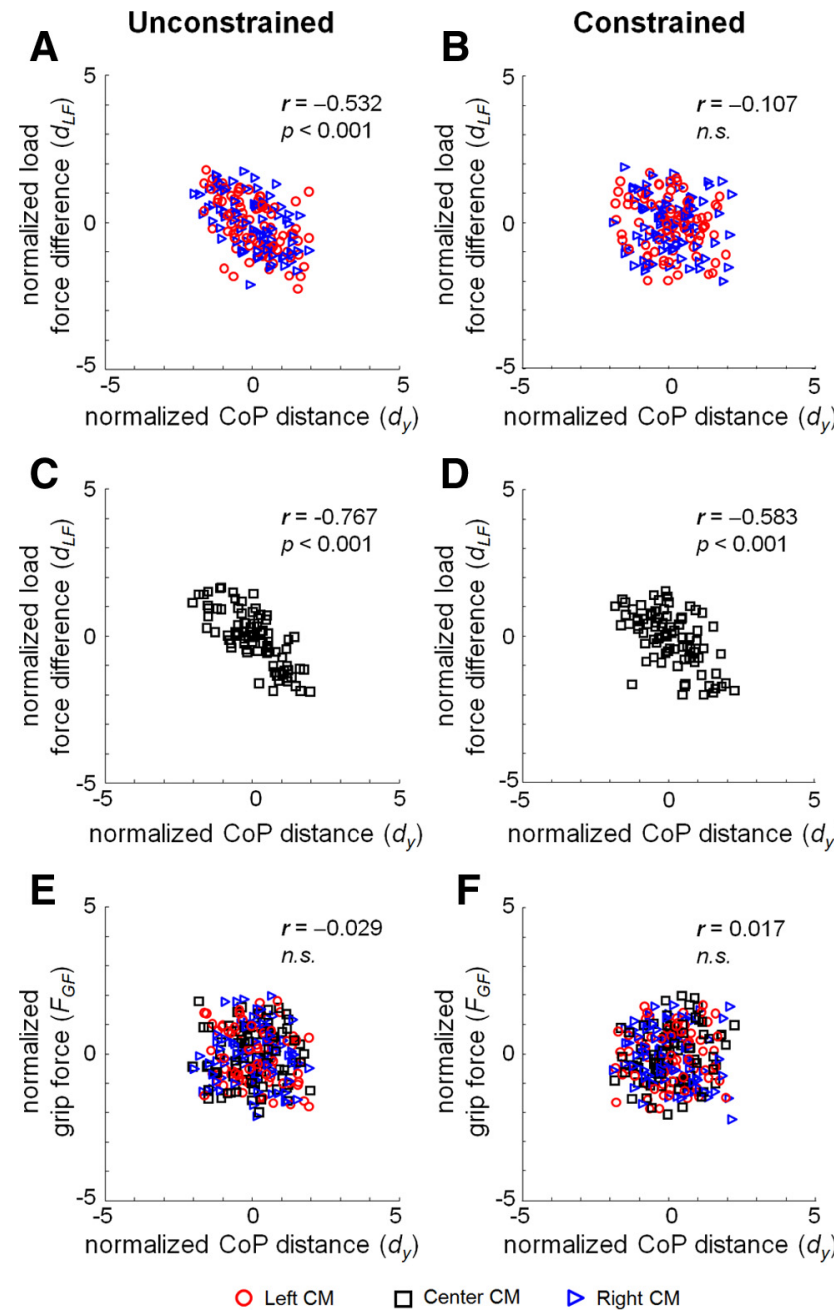

normalized CoP distance $\left(d_{y}\right)$

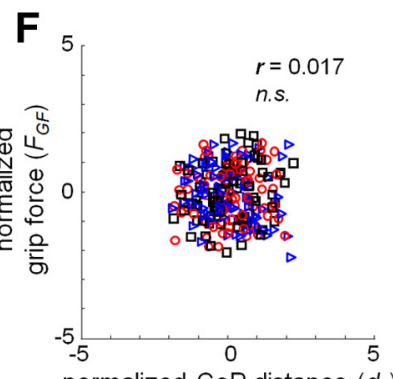

normalized CoP distance $\left(d_{y}\right)$

Center CM Right CM

Figure 8. Relations between digit centers of pressure, grip force, and load force. $\boldsymbol{A}, \boldsymbol{B}, d_{y}$ versus $d_{\mathrm{LF}}$ from the left and right $\mathrm{CM}$ conditions for unconstrained $(\boldsymbol{A})$ and constrained $(\boldsymbol{B})$ subject groups. $\boldsymbol{C}, \boldsymbol{D}, d_{\mathrm{y}}$ versus $d_{\mathrm{LF}}$ from the center $\mathrm{CM}$ condition for the unconstrained $(\boldsymbol{C})$ and constrained $(\boldsymbol{D})$ subject groups. $\boldsymbol{E}, \boldsymbol{F}, d_{\mathrm{y}}$ versus grip force for the unconstrained $(\boldsymbol{E})$ and constrained $(\boldsymbol{F})$ subject groups. Data are from trials $4-10$ from each subject and CM condition and are shown in normalized form. Pearson's $r$ value and corresponding $p$ value are shown in each panel. $p>0.05$. n.S., Not significant.

A major finding of this study was the inverse relation between the load forces applied by the thumb and index finger and the vertical spacing between the two fingers.

We found that removal of the digit placement constraints during the stable performance phase (trials 4-10) resulted in a larger digit spacing of the thumb and index finger (Fig. $5 A$ ), a more symmetrical sharing of load forces between these fingers (Fig. 6B), and smaller grip forces (Fig. 6A) than in the constrained group. These results suggest that subjects spontaneously chose to alter digit spacing and hand placement on the object and reduced the magnitude of grip forces and load force asymmetry. These data support our hypothesis that modulation of digit placement on objects contributes to optimal distribution of digit forces. This strategy might be associated with a smaller energy cost while avoiding implementation of largely asymmetric digit load forces, as well as prevent damage to the hand or object from excessive force. This behavior is reminiscent of force optimization implemented by humans exerting grip forces slightly above the minimum necessary to attain stable grasping (safety margin) (Johansson and Westling, 1984). Our findings suggest that sub 
jects are also able to optimize forces through an anticipatory adjustment in grasp posture.

The fact that the two CM conditions elicited different digit positions and force patterns in the unconstrained group suggests that factors other than force minimization may play a role in grasp planning. The asymmetric biomechanical capabilities of the two digits might also be important, allowing the thumb to exert a larger load force than the index finger (Salimi et al., 2000, 2003) and reducing the need for wide digit spacing in trials with the left CM.

\section{Variability in digit placement and covariation with load forces}

As predicted, a significantly larger variability in digit position was found when digit placement constraints were removed. Subjects might have been unable to store an accurate memory representation of the digit positions without visual cues provided by force sensors. Conversely, errors in reproducing a desired fingertip position might have resulted from errors in sensorimotor transformations (Soechting and Flanders, 1989). Psychophysical evidence, however, indicates that subjects have an accurate sense of fingertip position when matching object size by modulating finger span (Chieffi and Gentilucci, 1992; Santello and Soechting, 1997). Alternatively, subjects might have not tried to reproduce the same digit placement on each trial due to their ability to use a force compensation strategy.

It should be emphasized that similar trial-to-trial variability of compensatory torque was found in the constrained and unconstrained groups. This implies that subjects learned to compensate for digit placement variability through force modulation. These findings indicate that planning and execution of digit forces at object lift onset incorporated digit placement on a trial-to-trial basis. This is the first direct evidence that anticipatory force modulation on a given trial depends not only on sensorimotor memories of digit forces and positions from previous trials, but also on the actual position of each fingertip that requires afferent signals. Such position-dependent force modulation was not necessary to the same extent when the position variability was constrained by the grip device.

\section{Neural mechanisms underlying the concurrent modulation of digit placement and forces}

The attainment of a stable compensatory torque despite trial-totrial variability in digit placement and forces suggests the existence of a higher order motor plan that specifies the task goal independent of the different ways in which it can be reached, i.e., motor equivalence (Lashley, 1930; Raibert, 1977; Kelso et al., 1984; Cole and Abbs, 1986). It has been suggested that kinematic parameters are stored independently from the effectors used to perform a given task, the neural correlates residing in secondary and tertiary cortical areas including premotor dorsal cortex and middle intraparietal area (Rijntjes et al., 1999). However, to the best of our knowledge, no study has addressed how kinematics and kinetics are coordinated to attain motor equivalence in grasping. Here we propose a theoretical framework that accounts for our findings of digit position-dependent force modulation.

\section{Planning of digit positions and forces before contact}

Planning of digit positions and forces is thought to involve anterior intraparietal area (AIP), as indicated by the larger variability of digit placement and disruption of force coordination caused by transcranial magnetic stimulation of AIP $\sim 250$ and $\sim 150 \mathrm{~ms}$, respectively, before contact in grasp-to-lift tasks (Davare et al.,
2007). In our experiment, the unconstrained group could have planned digit positions based on digit placement used in previous trials as well as past experience (manipulating objects with similar properties). For the constrained group, physical landmarks determined the position of the digits by providing visual cues for digit position planning. Although monitoring of digit placement is likely driven by visual feedback, further work is needed to determine its role for contact point modulation.

With regard to digit force planning, previous grasp research constraining digit placement suggests that subjects quickly learn to program digit forces, as indicated by a stereotypical force development shortly after contact (Forssberg et al., 1991; Gordon et al., 1993). We speculate that some degree of digit force planning occurs also when digit placement is not constrained. However, our data suggest that such force planning might require a higher degree of online monitoring and corrections to compensate for the large variability of digit placement.

\section{Sensing of digit placement from contact to object lift onset}

Tactile afferents accurately encode information of contact timing, force direction, contact sites on the fingertip, and frictional condition in a very rapid fashion (Birznieks et al., 2001; Johansson and Birznieks, 2004; for review see Johansson and Flanagan, 2009). The extent to which tactile input contributes to sensing of the distance between digit centers of pressure is unknown. Nevertheless, this information is likely to be derived from integrating tactile input with visual and proprioceptive inputs.

\section{Digit position-dependent modulation of digit load forces}

For the unconstrained group, the vertical distance between digit centers of pressure at early contact closely resembled that found at object lift onset (Figs. 2, 5). This indicates that despite the fact that small changes in digit CoPs occur as forces are being exerted, the relative position of the fingertips at object lift onset was already defined shortly after contact. Following contact, a comparison is made between the expected (desired) and the actual feedback of digit placement. A mismatch would trigger a change in the planned digit forces and possibly update sensorimotor memories.

It has been suggested that force upgrades driven by discrepancies between expected and actual feedback involve primary motor cortex, inferior parietal cortex, and cerebellum (Jenmalm et al., 2006). Johansson and Flanagan (2008) have proposed a model for digit force control, based on comparing expected and actual sensory consequences of the motor plan at behaviorally crucial epochs of the task, i.e., sensorimotor control points. These comparisons may trigger corrective modulation of digit forces if a mismatch between planned and actual forces is sensed. Our findings extend this model by introducing several new elements, as follows: (1) sensing of digit position, (2) integration of sensed digit position with planning of digit forces, and (3) comparison between the expected sensory consequences associated with the desired versus sensed compensatory torque to correct digit force distributions at object lift onset.

The present results might lead to the improvement of computational models of how object manipulation is learned and controlled by introducing a new, yet crucial component in the study of grasping: coordination of digit placement and digit forces. We propose that the sensorimotor processes revealed here might account for our fundamental ability to manipulate objects despite variable digit positions in everyday grasping and tool use. 


\section{References}

Birznieks I, Jenmalm P, Goodwin AW, Johansson RS (2001) Encoding of direction of fingertip forces by human tactile afferents. J Neurosci 21: 8222-8237.

Burstedt MK, Flanagan JR, Johansson RS (1999) Control of grasp stability in humans under different frictional conditions during multidigit manipulation. J Neurophysiol 82:2393-2405.

Bursztyn LL, Flanagan JR (2008) Sensorimotor memory of weight asymmetry in object manipulation. Exp Brain Res 184:127-133.

Chieffi S, Gentilucci M (1993) Coordination between the transport and the grasp components during prehension movements. Exp Br Res 94:471-477.

Ciocarlie M, Dang H, Lukos J, Santello M, Allen P (2009) Functional analysis of finger contact locations during grasping. Paper presented at Third Joint EUROHAPTICS Conference and Symposium on Haptic Interfaces for Virtual Environment and Teleoperator Systems, Salt Lake City, March.

Cohen RG, Rosenbaum DA (2004) Where grasps are made reveals how grasps are planned: generation and recall of motor plans. Exp Brain Res 157:486-495.

Cole KJ, Abbs JH (1986) Coordination of three-joint digit movements for rapid finger-thumb grasp. J Neurophysiol 55:1407-1423.

Davare M, Andres M, Clerget E, Thonnard JL, Olivier E (2007) Temporal dissociation between hand shaping and grip force scaling in the anterior intraparietal area. J Neurosci 27:3974-3980.

Forssberg H, Eliasson AC, Kinoshita H, Johansson RS, Westling G (1991) Development of human precision grip. I. Basic coordination of force. Exp Brain Res 85:451-457.

Friedman J, Flash T (2007) Task-dependent selection of grasp kinematics and stiffness in human object manipulation. Cortex 43:444-460.

Gordon AM, Westling G, Cole KJ, Johansson RS (1993) Memory representations underlying motor commands used during manipulation of common and novel objects. J Neurophysiol 69:1789-1796.

Jenmalm P, Schmitz C, Forssberg H, Ehrsson HH (2006) Lighter or heavier than predicted: neural correlates of corrective mechanisms during erroneously programmed lifts. J Neurosci 26:9015-9021.

Johansson RS, Birznieks I (2004) First spikes in ensembles of human tactile afferents code complex spatial fingertip events. Nat Neurosci 7:170-177.

Johansson RS, Flanagan JR (2008) Tactile sensory control of object manipulation in humans. In: The senses: a comprehensive reference, Vol 6,
Somatosensation, (Gardner E, Kaas JH, eds), pp 67-86. San Diego: Academic.

Johansson RS, Flanagan JR (2009) Coding and use of tactile signals from the fingertips in object manipulation tasks. Nat Rev Neurosci 10:345-359.

Johansson RS, Westling G (1984) Roles of glabrous skin receptors and sensorimotor memory in automatic control of precision grip when lifting rougher or more slippery objects. Exp Brain Res 56:550-564.

Johansson RS, Westling G (1987) Signals in tactile afferents from the fingers eliciting adaptive motor responses during precision grip. Exp Brain Res 66:141-154.

Johansson RS, Westling G (1988a) Coordinated isometric muscle commands adequately and erroneously programmed for the weight during lifting task with precision grip. Exp Brain Res 71:59-71.

Johansson RS, Westling G (1988b) Programmed and triggered reactions to rapid load changes during precision. Exp Brain Res 71:72-86.

Kelso JA, Tuller B, Vatikiotis-Bateson E, Fowler CA (1984) Functionally specific articulatory cooperation following jaw perturbations during speech: evidence for coordinative structures. J Exp Psychol Hum Percept Perform 10:812-832.

Lashley K (1930) Basic neural mechanisms in behavior. Psychol Rev 37:1-24.

Lukos J, Ansuini C, Santello M (2007) Choice of contact points during multidigit grasping: effect of predictability of object center of mass location. J Neurosci 27:3894-3903.

Lukos JR, Ansuini C, Santello M (2008) Anticipatory control of grasping: independence of sensorimotor memories for kinematics and kinetics. J Neurosci 28:12765-12774.

Raibert MH (1977) Motor control and learning by the state space model. Technical report AI-M-351, Massachusetts Institute of Technology.

Rijntjes M, Dettmers C, Büchel C, Kiebel S, Frackowiak RS, Weiller C (1999) A blueprint for movement: functional and anatomical representations in the human motor system. J Neurosci 19:8043-8048.

Salimi I, Hollender I, Frazier W, Gordon AM (2000) Specificity of internal representations underlying grasping. J Neurophysiol 84:2390-2397.

Salimi I, Frazier W, Reilmann R, Gordon AM (2003) Selective use of visual information signaling objects' center of mass for anticipatory control of manipulative fingertip forces. Exp Brain Res 150:9-18.

Santello M, Soechting JF (1997) Matching object size by controlling finger span and hand shape. Somatosens Mot Res 14:203-212.

Soechting JF, Flanders M (1989) Errors in pointing are due to approximations in sensorimotor transformations. J Neurophysiol 62:595-608. 